\title{
Évacuation massive des populations en temps d'épidémie de COVID-19 : comment éviter la sur- crise?
}

Éric Daudé, Delphine Grancher et Franck Lavigne

\section{CpenEdition \\ Journals}

\section{Édition électronique}

URL : https://journals.openedition.org/echogeo/20961

DOI : $10.4000 /$ echogeo.20961

ISSN : 1963-1197

\section{Éditeur}

Pôle de recherche pour l'organisation et la diffusion de l'information géographique (CNRS UMR 8586)

Référence électronique

Éric Daudé, Delphine Grancher et Franck Lavigne, «Évacuation massive des populations en temps d'épidémie de COVID-19: comment éviter la sur-crise? », EchoGéo [En ligne], Sur le Vif, mis en ligne le 25 mars 2021, consulté le 24 août 2021. URL : http://journals.openedition.org/echogeo/20961 ; DOI : https://doi.org/10.4000/echogeo.20961

Ce document a été généré automatiquement le 24 août 2021.

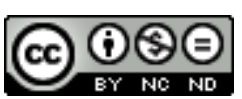

EchoGéo est mis à disposition selon les termes de la licence Creative Commons Attribution - Pas d'Utilisation Commerciale - Pas de Modification 4.0 International 


\title{
Évacuation massive des populations en temps d'épidémie de COVID-19: comment éviter la sur-crise?
}

\author{
Éric Daudé, Delphine Grancher et Franck Lavigne
}

Ce travail est réalisé dans le cadre du projet ANR ESCAPE soutenu par l'Agence Nationale de la Recherche, ANR-16-CE39-0011-01.

1 La pandémie de COVID-19 qui dure depuis près d'une année est une crise majeure du fait de ses retombées sanitaires, sociales et économiques. Elle nécessite la pleine concentration des services de soins, ce qui au moment de la première vague a engendré un recul de l'attention portée à des nombreuses autres pathologies ou maladies dont les conséquences se mesureront à moyen et long terme. Elle mobilise également pleinement la vigilance et les ressources économiques des États, des organisations, des entreprises et des individus pour y faire face. Cette focalisation nécessaire pour tenter de limiter au mieux les effets et revenir au plus vite à une « vie ordinaire » a engendré une exposition accrue à d'autres aléas. Or la durée de cette pandémie ne peut que créer des situations multi crise, du fait de la périodicité prévisible de certains aléas, tels que les aléas naturels. Parmi ceci, on pense notamment aux cyclones et aux inondations qui chaque année sont à l'origine de pertes humaines et de dégâts matériels considérables. Or comment faire face à un cyclone et ses conséquences lorsque toute l'attention se focalise sur la pandémie? Comment concilier des stratégies de luttes organisées dans une vision mono-aléa lorsque la situation nécessiterait de penser des stratégies multi risques qui présentent potentiellement des situations contradictoires. L'objectif de ce papier, à travers la présentation d'exemples de situations multi crise qui se sont produits durant l'année 2020, est de faire un point sur les enjeux, les verrous et les défis à relever dans un tel contexte. 


\section{Le talon d'Achille de la gestion des risques : la réduction des risques de catastrophes multi-aléas}

2 Une crise est le dysfonctionnement d'un système qui résulte d'une perturbation. Celleci met potentiellement en danger le fonctionnement des organisations qui le structurent et peut conduire à la catastrophe. La gestion d'une crise consiste donc à limiter les effets potentiels de ces perturbations sur les territoires. Elle se décline en plusieurs volets qui mobilisent des compétences et des outils différents, qui impliquent communications et actions pour protéger la population (Leone et al., 2010; Sauvagnargues 2019). Cet arsenal de moyens inclut notamment des systèmes de surveillance, des procédures d'alerte à la population, des consignes de sécurité, de préparation, de protection et de mise à l'abri (Bundy et al., 2017). Parmi celles-ci, l'évacuation massive est l'une des stratégies qui peut être envisagée pour écarter d'une zone de danger la population, pour quelques heures, quelques jours ou plusieurs semaines via l'utilisation de centres de refuge ou de véritables camps de déplacés (CEPRI, 2014 ; Esposito Amideo et al., 2019).

3 Les plans de gestion des crises qui ont pour objectif la préparation des services et des territoires, ce qui comprend les consignes à donner à la population ainsi que la délimitation des zones exposées, prennent cependant rarement en compte les risques de sur-crise. Par sur-crise, nous entendons une amplification de la crise initiale en lien soit avec l'occurrence d'un nouvel aléa (un tsunami après un tremblement de terre) soit des effets systémiques avec des défaillances en cascade au sein de l'organisation de la gestion de crise (une épidémie et une défaillance du système de soin). L'une des causes possibles de sur-crise est que ces plans sont le plus souvent fondés sur une approche aléa-centrée et mono-aléa : plan inondation, plan submersion marine, plan mouvement de terrain, plan éruption volcanique, etc. Ils semblent ainsi plus faciles à élaborer et à mettre en œuvre car ils ne prennent justement pas en compte la complexité des interactions entre plusieurs aléas et leur gestion, ni n'impliquent une géographie évolutive de la crise. Ce qui peut amener à une sur-crise. Or, de nombreux territoires sont exposés à différentes menaces naturelles non directement liées, telles les Antilles ou les Philippines qui sont exposées aux aléas cycloniques, sismiques et volcaniques (illustration 1). 


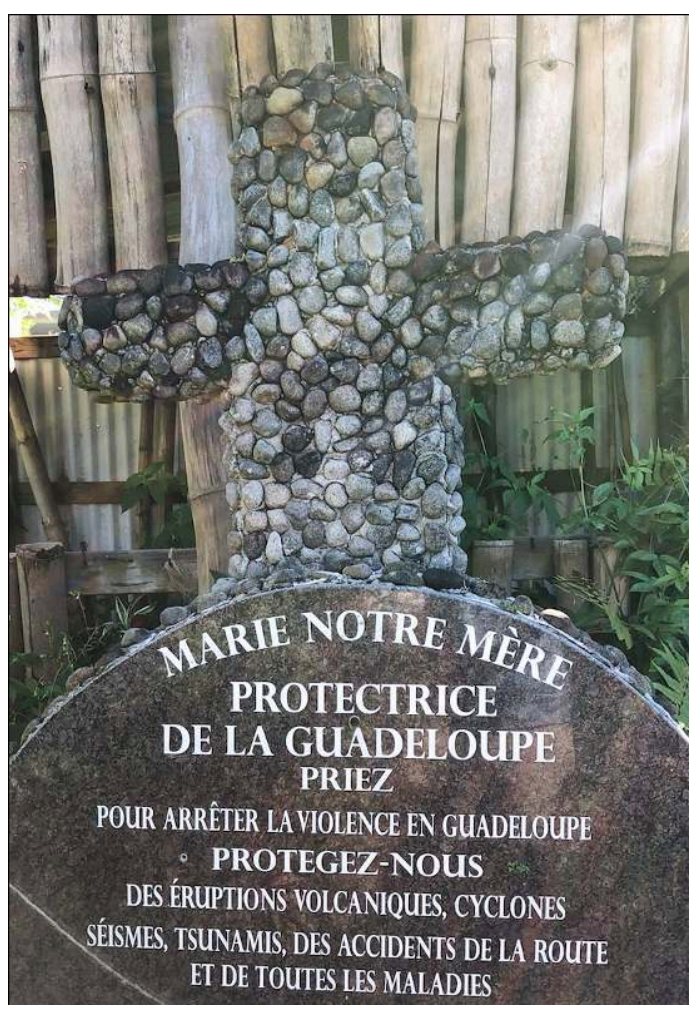

Auteur : É. Daudé.

4 Même si la coexistence de plusieurs risques peut être communiquée à la population dans un document unique, par exemple en France le Document d'Information Communal sur les Risques Majeurs (DICRIM), les plans de gestion de crise définis par les autorités demeurent généralistes ou déclinés pour chacun des aléas. Les consignes ou décisions prévues ne sont donc non seulement pas hiérarchisées pour privilégier l'une ou l'autre des contraintes, ni pour les autorités ni pour les populations, en cas d'aléas d'origine différente et simultanée, ni pensée en termes de complémentarités ou de contraintes entre elles dans un contexte de sur-crise. Dit autrement, le risque systémique est trop peu souvent envisagé dans les plans et la préparation à la gestion de crise.

5 Des retours d'expériences menés au lendemain de catastrophes multi-aléas ont malgré tout conduit à la mise en place de stratégies et de procédures spécifiques pour anticiper les effets domino prévisibles et ainsi limiter les risques de sur-crise (Shaw et al., 2013; Few et Matthies, 2006). Cependant, force est de constater que ces initiatives sont rares et que la gestion au coup par coup, avec une vision à court terme en fonction de la chronologie des événements, reste la pratique la plus répandue. Ce fut par exemple le cas aux Philippines, à la suite de l'éruption du volcan Pinatubo en 1991, lorsque des lahars (coulées de débris et de boues d'origine volcanique) dévastateurs furent provoqués par un cyclone (Janda et al., 1996). Comme l'ont aussi montré les catastrophes liées aux séismes suivis de tsunamis de Tohoku (Japon) en mars 2011 ou à Palu (Indonésie) en octobre 2018 (Omira et al., 2019), la sur-crise ne prolonge pas simplement la crise, mais constitue un état modifié de celle-ci, qui résulte d'une nouvelle perturbation contradictoire aux connaissances et/ou aux outils déployés 
jusque-là pour réduire les effets de la crise initiale. C'est précisément le cas lorsqu'une catastrophe d'origine naturelle intervient dans un contexte de pandémie.

\section{Gestion de crises d'origine naturelle en contexte de pandémie}

6 L'Histoire nous a montré que de grandes catastrophes naturelles ont souvent été à l'origine d'épidémies voire de pandémie. Les éruptions volcaniques majeures provoquent systématiquement des pertes de récoltes catastrophiques qui entrainent des famines. Ces événements en cascade sont le plus souvent engendrés à l'échelle régionale par l'impact direct des cendres, comme en 1815 sur les iles indonésiennes de Sumbawa et Lombok suite à l'éruption du volcan Tambora. Les famines et les épidémies apparaissent également à la suite de perturbations climatiques engendrées par les éruptions à l'échelle globale, comme illustré en Europe et au Japon suite à l'éruption du volcan indonésien Samalas en 1257 (Guillet et al., 2017). Les cyclones dévastateurs du $\mathrm{XX}$ siècle ont aussi été à l'origine d'épisodes épidémiques : en Haïti, des épidémies de choléra se sont propagées à plusieurs reprises après des cyclones dévastateurs (Piarroux, 2019).

7 Certaines catastrophes d'origine naturelle se sont aussi produites pendant une période de pandémie. Au lendemain de la Première Guerre Mondiale, la "grippe espagnole » a ainsi fragilisé des dizaines de millions de personnes en raison de sa souche particulièrement virulente et contagieuse (H1N1). Les effets de l'intense sècheresse due au El Niño de 1918-1919, considéré comme l'un des plus puissants du XX ${ }^{\mathrm{e}}$ siècle (Giese et al., 2010), ont probablement contribué aux 18 millions de morts en Inde de ce virus (Dye et al., 1988)

Cette concomitance d'événements majeurs a des conséquences en termes de gestion de crise, notamment du fait de la segmentation des compétences selon la nature de l'aléa et d'une emprise territoriale des aléas pouvant être disjointes en partie. En Chine, le coronavirus responsable du syndrome respiratoire aigu sévère (SRAS) a émergé puis s'est propagé entre novembre 2002 et juillet 2003 dans trente pays. Cette pandémie a mis en évidence que sur de nombreux territoires ce n'étaient pas les mêmes acteurs qui géraient les risques sanitaires et les risques naturels (Zhang, 2012). Or la coordination entre tous les acteurs se complexifie en l'absence de préparation à des changements de doctrine en cours de crise. Ainsi l'épidémie du SRAS de 2013 a permis de modifier le schéma initial de gestion des crises en Chine, dans le but de clarifier les responsabilités des gouvernements centraux et locaux, ainsi que des ministères et des agences.

Que ce soit des événements quasiment synchrones dans des espaces restreints ou à l'échelle nationale, ces catastrophes illustrent donc les difficultés du contexte multi risques et invitent à penser l'harmonisation des procédures, l'anticipation et la préparation à l'aggravation des crises par la survenue d'une autre crise.

\section{L'analyse de la pandémie de COVID-19 pour questionner la gestion des risques naturels et sanitaires simultanés}

La période actuelle, marquée par la propagation de la COVID-19 est particulièrement compliquée pour tous les acteurs de gestion de crises qui doivent, en plus de gérer la pandémie, se préparer à d'autres événements potentiellement catastrophiques. Des cyclones, des inondations graves ou des séismes qui ont nécessité des évacuations de 
masse ont déjà eu lieu depuis le début de la pandémie et se renouvelleront du fait notamment de leur saisonnalité sur certains territoires (c'est le cas en particulier des cyclones). Prendre en compte ce contexte sanitaire dans la gestion de cet évènement amène des difficultés supplémentaires.

11 En l'absence de vaccin près d'un an après le début de la pandémie et jusqu'au début de 2021, la stratégie privilégiée par presque tous les territoires pour lutter contre la progression du virus et faire barrage aux vagues épidémiques a été de réduire voire interdire les déplacements et les rassemblements. Les fermetures des frontières et la limitation des transports se sont ajoutées à un confinement plus ou moins strict de la population : universités, salles de sport, théâtres, cinémas et commerces non essentiels fermés, incitation au télétravail, couvre-feu etc. Ces restrictions se sont accompagnées de mesures individuelles de précaution - port du masque, distanciation physique, lavage des mains - imposées dans certaines circonstances (dans les transports publics, les établissements scolaires, les commerces etc.). Les autorités, les entreprises, les institutions et les populations doivent donc depuis plusieurs mois anticiper et organiser leurs activités au rythme des consignes successives de confinement et de déconfinement imposées pour lutter contre la progression de l'épidémie. Si cette situation fragilise l'économie des territoires et les systèmes de santé, elle fragilise encore plus durement les populations les plus vulnérables ${ }^{1}$ et l'hypothèse de la survenue d'une autre crise constitue un véritable défi pour nos sociétés.

Or cette situation est certaine dans de nombreux territoires et pour ceux où l'évacuation des populations est prévue dans les plans de gestion de crise. Les autorités doivent donc établir un état des lieux des difficultés que pourrait faire peser la pandémie sur cette stratégie. En effet, comment inciter les populations à quitter leur domicile et à évacuer une ville, comment gérer leurs déplacements en particulier s'ils doivent être massifs et rapides, comment adapter les hébergements collectifs lorsque toutes les mesures prises jusqu'à présent pour limiter la diffusion du virus se sont focalisées sur le confinement (rester chez soi), la restriction des déplacements et la distanciation physique? Parmi toutes les étapes d'une gestion de crise, l'évacuation massive est donc surement celle dont l'adaptation à cause de la COVID-19 présente le plus d'enjeux (Quigley et al., 2020). Et les effets potentiels de ces deux stratégies opposées - évacuer en période de confinement ou de distanciation physique - sont encore peu anticipés et étudiés étant donné l'aspect inédit de cette pandémie.

\section{Objectifs et méthode}

13 Le présent article a pour principal objectif de décrire et de lancer des pistes de réflexion sur les principaux problèmes posés ou qui risquent de se poser en cas de surcrise de la pandémie liée à un aléa naturel. À partir de principes théoriques et d'exemples précis, nous détaillons les conséquences possibles ou déjà observées des consignes sanitaires strictes liées à la pandémie actuelle sur les évacuations de populations exposées à une menace. Nous nous intéressons ici exclusivement au cas de crises d'origine naturelle en focalisant un peu plus nos propos sur la zone intertropicale, dont l'exposition et la vulnérabilité des populations sont un enjeu particulièrement important. Plus précisément, cet article a été construit à partir de rares articles scientifiques sur la question, d'articles de presse dans la mesure où cette crise est très récente et toujours en cours et d'informations et réflexions issues d'une 
expertise plus approfondie sur certaines zones géographiques connues par les auteurs (Indonésie, Inde, Antilles, etc.), notamment concernant l'adaptation des plans de gestion des risques et des crises d'origine naturelle.

Nous illustrons notre réflexion avec deux cyclones de catégorie 5 sur l'échelle de SaffirSimpson et deux séismes, ainsi que l'étude de la documentation préventive distribuée en Indonésie et à Sint-Maarten (Antilles). Les deux cyclones sont d'abord Harold qui a ravagé début avril 2020 le Vanuatu (27 victimes), avec des pointes de vent de $230 \mathrm{~km} / \mathrm{h}$. Cet exemple permettra en particulier de soulever les questions d'entraide internationale et du rôle des ONG. Le second est le cyclone Amphan qui a balayé la région de Calcutta et le Bangladesh en mai 2020. Ce cyclone est le plus violent survenu dans le Golfe du Bengale depuis 2007. Les vents violents (jusqu'à $240 \mathrm{~km} / \mathrm{h}$ ), les pluies diluviennes et une submersion marine de près de 3,5 mètres ont entrainé la mort de 118 personnes. Ce super cyclone a nécessité l'évacuation de plus de 2 millions de personnes dans un contexte sanitaire et médical particulièrement fragile. Enfin les séismes de Zagreb de magnitude 5.3 du 22 mars 2020 et de l'État d'Oaxaca au Mexique de magnitude 7.5 du 23 juin 2020 (6 morts) ont directement illustré les difficultés de l'évacuation spontanée des populations dans des territoires soumis à un confinement.

\section{Coordonner les acteurs de la gestion de crise}

La gestion des risques naturels est structurée dans chaque État selon des organigrammes prédéfinis, souvent très hiérarchisés, qui permettent d'attribuer à chaque service les responsabilités d'organisation des secours et de la logistique. Dès le début de la crise sanitaire mondiale (la COVID-19 a été déclarée pandémie le 11 mars 2020 par l'OMS), les États ont mobilisé des ressources humaines, matérielles et financières de différents services : ministère de la Santé bien sûr, mais également de la sécurité civile, de l'armée ou encore des transports pour contenir la maladie. Les réponses stratégiques ont varié d'un pays à l'autre. Après une période d'ajustement liée au manque de ressources pour opérationnaliser la stratégie (manque de masques, de tests, hôpitaux sous-dimensionnés), les différents acteurs mobilisés dans la gestion de cette épidémie sont parvenus dans de nombreux pays à ralentir en deux ou trois mois la première vague, alors que d'autres déployant d'autres stratégies n'y sont pas parvenus. Quelle que soit la situation aujourd'hui, avec de nombreux pays touchés par une seconde vague, une nouvelle crise liée à un aléa de nature différente constitue un véritable défi stratégique et opérationnel.

La superposition d'un nouvel évènement majeur provoque en effet une " concurrence " entre les plans de secours prévus pour lutter contre ce nouvel aléa et ceux de la gestion de crise sanitaire en cours, notamment sur les aspects tactiques et opérationnels. Chaque plan est conçu pour un seul aléa indépendamment des autres, il ne prend donc pas en compte les acteurs qui seraient déjà mobilisés. Si cette conjonction conduit inévitablement à mobiliser les mêmes acteurs (les hôpitaux par exemple), elle amène aussi potentiellement son lot de nouveaux acteurs (ONG, armées) et pose des contraintes importantes pour le déploiement des secours.

17 À l'échelle internationale, l'aide humanitaire et les ONG d'urgence classiquement mobilisées lors des catastrophes majeures sont ainsi déjà considérablement paralysées. Les fermetures de frontières décidées généralement unilatéralement dans un contexte officiel d'état d'urgence national pour empêcher l'arrivée de personnes contagieuses ne 
sont pas facilement dérogeables, même pour favoriser le soutien international. Il s'agit en effet pour les autorités de préserver au maximum leur territoire déjà meurtri d'une contamination croissante qu'ils ne pourraient gérer. En milieu insulaire et en cas de soutien de forces extérieures par exemple, il est possible que les territoires ou pays imposent une quarantaine à ces personnels. Les militaires de l'opération Résilience en France ont ainsi été soumis à une quarantaine avant de débarquer sur les territoires outre-mer français où ils étaient attendus en renfort des hôpitaux locaux au printemps 2020. Cette étape de quarantaine a ralenti la mise en place du soutien pour la COVID-19, et si une autre opération devait être déclenchée en cas de séisme ou de cyclone sur un des territoires ultramarins français, la même organisation (envoi de forces militaires extérieures au territoire) ne pourrait être rapidement opérationnelle sans que ces forces se soumettent antérieurement à un isolement complet. Autre exemple, au Vanuatu, où les frontières étaient fermées pour cause de pandémie au moment du passage du cyclone Harold, l'acheminement de l'aide humanitaire a été largement perturbé. D'origine étrangère, en particulier via les ONG internationales, il a souffert d'un retard conséquent obligeant les autorités nationales à gérer seules les secours à la population ${ }^{2}$.

18 À l'échelle nationale, la mobilisation de ressources matérielles et humaines pour soutenir des territoires dont le système de santé est en tension à cause de la CoVID-19 limite forcément un déploiement immédiat en cas de nouveaux besoins et réduit la disponibilité des moyens. C'est ce qu'on a pu observer en Odissa (Inde) où la coordination entre les services de santé, les autorités locales et la force d'intervention en cas de catastrophe ${ }^{3}$ a pu être problématique. En effet, nombre d'abris anticycloniques usuels avaient déjà été réquisitionnés pour servir de centres de quarantaine pour des malades de la COVID-19 alors gérés par les services de santé avant l'arrivée du cyclone Amphan (Boyland, Adelina, 2020). Cette situation a conduit à des adaptations contextuelles entre d'un côté la NDRF, les Panchayats (gouvernements locaux villageois) et les institutions qui ont pu proposer des solutions alternatives face au danger. Cette décentralisation de la décision et de l'action est d'autant plus nécessaire que c'est à un niveau local que la connaissance des populations vulnérables, de l'éducation aux dangers et du territoire est la plus concrète. Ces négociations au niveau local ont alors permis à de nombreux villages de choisir des abris alternatifs à ceux proposés par les autorités indiennes pour se protéger du cyclone Amphan ${ }^{4}$.

Paradoxalement, la mobilisation pour renforcer les services de soin peut constituer également un avantage pour la gestion des autres risques. Ainsi la France en mobilisant des forces militaires pour aider au transfert des malades et renforcer les équipes médicales locales lors de l'opération Résilience en outre-mer Français a aussi renforcé la préparation des territoires à la saison cyclonique à venir (juin-octobre) en effectuant des exercices de reconnaissance. Un autre effet relativement positif est la présence déjà opérationnelle des ONG médicales locales ou internationales en intervention pour soulager le système de santé et sensibiliser la population (par exemple Médecins sans Frontières en Haïti en mai 2020). On peut supposer leur déploiement rapide en cas de catastrophe d'origine naturelle. Malgré ces derniers exemples très spécifiques, l'importance d'une vérification globale de la capacité de réaction des acteurs potentiellement impliqués dans une gestion de crise, et leur disponibilité, est indispensable. 
Il s'agit en effet de protéger les intervenants du secours et du soutien aux déplacés. La protection des personnes extérieures doit être garantie, notamment les plus âgées (souvent actives sous la forme de bénévolat) dont on connait la vulnérabilité plus grande à la COVID19 (Nikolich-Zugich et al., 2020). On peut anticiper une réticence de leur part à vouloir intervenir dans les zones avec de fortes densités ou à être en contact avec beaucoup de personnes différentes dans des conditions d'hygiène non garantie. Ce cas s'est présenté au Japon lors des fortes inondations en juillet 2020 qui ont nécessité l'évacuation de plus de 450000 personnes sur l'ile de Kyushu'. Ces exemples posent la question des effectifs mobilisables en gestion de sur-crise et de leur vulnérabilité. Ceci suppose d'évaluer à la fois les forces vives à disposition malgré la mobilisation pour la gestion de la COVID-19 et les possibles refus d'action liés au choix de protection des intervenants et de leurs proches au détriment de l'engagement professionnel ou volontaire. Avec la double crise épidémie et aléa naturel, ce phénomène peut ne pas être marginal et donc peser sur la conduite des plans.

\section{Gérer de nouvelles répartitions démographiques, redimensionner les abris, prendre en charge les malades}

21 On l'a vu avec les personnels professionnels ou bénévoles, la gestion de crise soulève des enjeux logistiques pour les autorités et pour les populations. Lorsque l'épidémie est présente sur un territoire, le nombre de personnes sous respirateur artificiel est supérieur à la capacité habituelle des unités locales, car les formes les plus graves de la COVID-19 entrainent des complications qui nécessitent souvent un long séjour, jusqu'à plusieurs semaines, en service de réanimation. Or l'étape la plus critique d'une évacuation de masse classique concerne les personnes hospitalisées (Duanmu et al., 2010) et les personnes non-autonomes (sans véhicule personnel). Une zone à évacuer qui serait touchée par la COVID-19 nécessitera donc d'importantes ressources supplémentaires pour assurer le transfert de ces malades. Ils seront transférés vers d'autres établissements dans des conditions particulières complexes, notamment pour les personnes en réanimation qui nécessitent la disponibilité d'équipements spécifiques dans les hôpitaux d'accueil. En cas d'alerte tsunami, l'UNESCO a donc rédigé un guide synthétisant les mesures à prendre pour évacuer les personnes affectées par la COVID-19, incluant le personnel soignant (UNESCO, 2020). La survenue d'un évènement majeur peut aussi directement affecter les structures de soins, dont celle des malades COVID-19. Le typhon Vongfong (mai 2020) a par exemple détruit les deux tiers des lits de quarantaines destinés aux malades de la COVID-19 dans la province du Samar Oriental aux Philippines'.

Dans le cas des cyclones, la phase d'alerte est associée à la préparation des abris par les autorités. Quel que soit l'aléa pris en compte, les plans de gestion sont adaptés au contexte démographique de la zone sur laquelle ils s'appliquent, en anticipant les moyens médicaux mobilisés et leur décharge en cas d'afflux de blessés (Jassempour et al., 2014), en prévoyant des espaces (généralement publics) équipés pour l'hébergement et la nourriture ou en organisant des jumelages entre communes (Elysia et Wihadanto, 2018). 
Or cette crise met en évidence l'interdépendance et les effets en cascade de risques complexes entre les systèmes. Ainsi le confinement national ou régional a pu modifier la répartition démographique dans de nombreux pays, en perturbant l'équilibre villes campagnes par exemple et en changeant les caractéristiques socioéconomiques des populations locales (Valsecchi et Durante, 2020). Les populations urbaines ont en effet parfois massivement quitté les grandes agglomérations pour les campagnes. Par exemple en Inde, des centaines de milliers de travailleurs ayant perdu leur emploi ont été contraints de revenir dans leurs villages d'origine (Bercegol et al., 2020). En Malaisie, ce sont les habitants de plusieurs villages qui se sont réfugiés dans les forêts par crainte d'être contaminés par la COVID-19, devenant malgré eux le vecteur de la COVID-19 dans ces zones de refuge ${ }^{7}$. Ces départs non anticipés par les gouvernements nationaux ou locaux ont augmenté les populations dans certains territoires sans aucune adaptation des infrastructures de santé ou d'hébergement. Dans des zones exposées aux aléas naturels, ce déséquilibre brutal a mis alors en danger la logistique de crise prévue par les autorités, d'autant plus que ces nouvelles populations étaient plus touchées par le virus que les provinces reculées, la COVID-19 étant principalement urbaine au début de l'épidémie. Pour gérer l'arrivée massive de cette population et contenir la propagation du virus sur leur territoire, les autorités sanitaires ont donc dû placer une partie de ces migrants spontanés en quarantaine et isoler ceux qui étaient déjà malades. Il a donc été nécessaire de trouver très rapidement des solutions d'hébergement adaptés à recevoir du public. La solution pragmatique a été d'utiliser les abris officiels destinés à héberger les populations en cas d'alerte, notamment cyclonique. C'est la stratégie qui a été choisie au Bengale-Occidental et en Odissa. Lorsque le cyclone Amphan est arrivé, ces réfugiés placés en quarantaine, les malades de la COVID-19 et les personnes fuyant le cyclone se sont alors retrouvés mélangés dans les mêmes abris ${ }^{8}$. Cette problématique d'abris sur peuplés s'est reproduit au Vietnam lors du passage du cyclone Molave qui a conduit plus de 375000 personnes à être évacuées vers des centres d'accueils en octobre 2020 (UN, 2020). Afin d'éviter ces situations et pour limiter le nombre de personnes initialement prévu par abri, il est nécessaire de réquisitionner un plus grand nombre de bâtiments, comme des écoles, des bâtiments publics ou encore des entreprises. Ainsi au Bangladesh, alors que 4500 abris pouvant accueillir chacun entre 600 et 1200 personnes étaient initialement prévus et disponibles dans la zone touchée par le cyclone Amphan, ce sont plus de 12000 abris qui ont dû être ouverts pour accueillir les personnes évacuées et respecter un seuil maximal de densité par centre ${ }^{9}$. 
Illustration 2 - Abri, distanciation physique et mesure de protection individuelle dans un abri lors du passage du cyclone Amphan

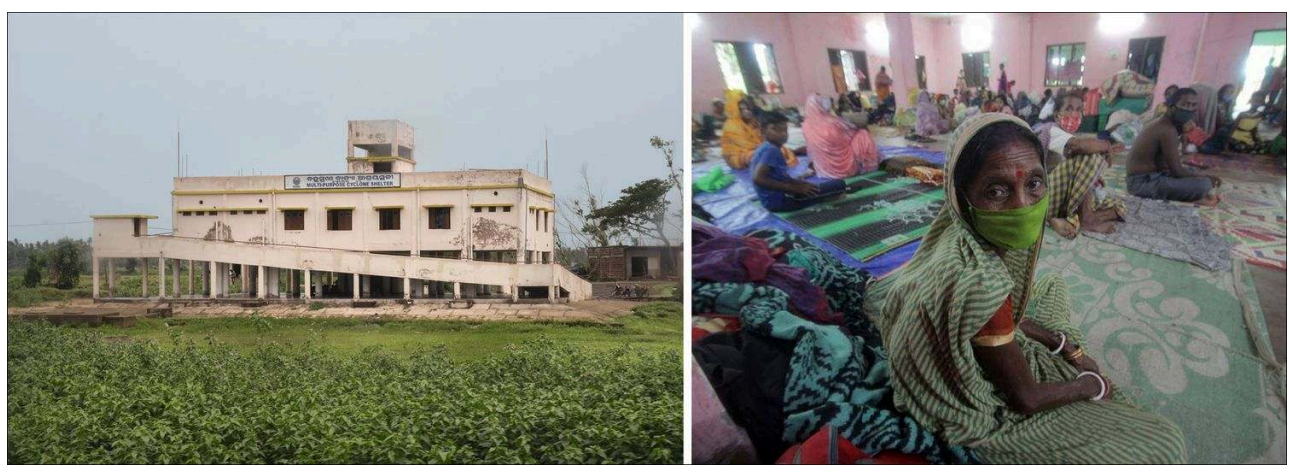

droite : https://www.thenewhumanitarian.org/analysis/2019/07/04/india-mass-cyclone-evacuationssaved-lives-not-livelihoods - gauche : https://www.canberratimes.com.au/story/6762875/cyclonedumps-rain-on-india-bangladesh/?cs=14264

À l'intérieur des abris, il est également nécessaire de limiter au maximum la propagation du virus et gérer les malades, en particulier les cas les plus graves. D'abord il s'agit de détecter (thermomètres, tests) et d'isoler les malades parmi ces populations hébergées collectivement. Cependant, malgré les efforts des autorités pour aménager les refuges et garantir la sécurité sanitaire, certaines populations n'accepteront pas l'ordre d'évacuation. La crainte de voir apparaitre des foyers de contamination dans des abris surpeuplés est réelle ${ }^{10}$. Elle pousse de nombreuses personnes à refuser de se rendre dans les abris, comme lors des inondations au Japon où de nombreuses personnes ont préféré s'abriter à l'intérieur de leur voiture au détriment de leur sécurité ${ }^{11}$. Ce comportement pourtant dangereux met en exergue un vrai dilemme entre l'évacuation et la protection face à l'épidémie pour les populations.

En effet, alors que pour un certain nombre d'aléas naturels les consignes officielles incitent à quitter son logement et à se regrouper collectivement dans des endroits ciblés (en hauteur dans un bâtiment, en altitude ou loin du rivage), les gestes barrières et les restrictions de déplacement stipulent de limiter les contacts et de ne pas s'éloigner du domicile dans le cadre de la lutte contre la COVID-19. Et dans cette situation de pandémie, les populations sont abondamment « exposées » à des messages privilégiant la prévention sanitaire. Le «nouveau » risque qui se présente pour chaque personne est donc à évaluer par rapport à la crainte préalable de tomber malade ou de contaminer un proche fragile et non par rapport à une situation sanitaire normale. La situation sanitaire qui dure depuis plusieurs mois et qui a fragilisé économiquement et psychologiquement des franges de la population ${ }^{12}$ renforce cette difficulté. L'annonce d'un nouvel aléa peut donc être encore plus difficile à gérer psychologiquement, la prise de décision devenant encore plus problématique (Cozic et al., 2020). Pour les populations et notamment les plus vulnérables, en plus d'abandonner ses biens et son domicile, c'est donc un dilemme qui se présente: fuir un danger plus ou moins imminent et connu mais risquer d'être contaminé par un virus potentiellement mortel. Selon l'état de santé, la qualité du logement, les ressources, mais aussi sa perception du danger, la résolution du dilemme demande une priorisation de l'un ou l'autre des deux aléas. En situation de pandémie, les familles doivent dès lors revoir toute leur "stratégie » afin de préserver les personnes les plus fragiles face au virus mais au détriment potentiel de leur sécurité face à l'aléa. Pour les populations des zones d'accueil, l'hospitalité fut ou devrait être aussi un dilemme qu'il va falloir résoudre 
individuellement ou collectivement: en Indonésie, certains villages ont rajouté des barrières à l'entrée pour interdire l'accès à toute personne non résidente (Widianto et al., 2020), pouvant alors constituer un véritable verrou à une évacuation spontanée.

Une autre difficulté vient de la capacité d'anticipation des populations. Les populations situées dans les zones exposées aux cyclones ont pour habitude et consigne de se préparer quelques jours avant l'arrivée d'un cyclone, et ceci passe par l'achat d'équipements de protection et de première nécessité. Chacun renforce son habitation et fait des réserves de nourriture et d'eau. Ces comportements "réflexes" reposent sur l'expérience des cyclones passés mais aussi sur le rappel de consignes dans les journaux ou par des ONG. Réalisées par des dizaines ou des centaines de milliers de personnes, ces préparations entrainent classiquement des ruées massives dans les supermarchés pour l'approvisionnement en matériel de protection de l'habitat et en produits alimentaires. Mais en situation d'épidémie, ces situations de forte affluence doivent être évitées au maximum et peuvent même être interdites (nombre de personnes limité dans les commerces par exemple). La spécificité du contexte sanitaire fragilise donc le bon déroulement de cette phase préparatoire. Certains produits ont été rapidement en rupture de stock dès le début du confinement dans tous les pays et on a pu observer des scènes de désordre public ainsi que de longues files d'attente ${ }^{13}$. La pénurie de produits d'hygiène (gel, masque, savon...) risque d'être amplifiée par les recommandations pourtant indispensables de les rajouter dans le kit de secours préparatoire par exemple à la survenue d'un tsunami, comme c'est le cas en Indonésie (UNESCO, 2020) ou d'un cyclone en début de saison cyclonique comme en Floride ${ }^{14}$. Ainsi une anticipation de la consommation particulière due à la fois au contexte pandémique qui serait concomitant avec une alerte cyclonique est indispensable afin d'éviter des pénuries, d'autant plus si le pays se trouve dans une phase de confinement avec un arrêt complet de son économie. La question des moyens logistiques doit donc aussi être soulevée. L'évacuation des populations au Bangladesh a ainsi été ralentie du fait d'une grave pénurie des transports locaux et de l'approvisionnement en essence, en raison des intempéries et de l'arrêt de toutes les activités économiques dû au lock down ${ }^{15}$.

L'anticipation des consignes et la préparation des populations devraient permettre de réduire les effets $\mathrm{du}$ stress sur les populations et d'anticiper ces réactions problématiques. Le gouvernement de Sint-Maarten aux Antilles a par exemple demandé aux populations dès le mois de mai 2020 de s'inscrire pour réserver les places dont elles ont besoin, ceci en amont de la saison cyclonique qui s'écoule de juin à octobre, et en prévision de la diminution des capacités d'hébergement des refuges publics. Sans connaitre l'évolution de l'épidémie des prochains mois sur leur île, les habitants ont donc pu anticiper et choisir une stratégie de refuge. La préparation des populations à la concordance des deux phénomènes peut permettre d'obtenir un compromis permettant une acceptation plus facile de l'évacuation, et de l'hébergement collectif, malgré une exposition accrue au virus.

\section{Conclusion}

Comment évacuer des centaines de milliers voire des millions de personnes en période d'épidémie et sous la contrainte d'un confinement? En privilégiant la menace imminente de destruction que fait peser par exemple un cyclone majeur aux conséquences possibles d'une évacuation sur la dynamique de l'épidémie, il est en effet 
indispensable de s'adapter aux nouvelles contraintes. Les cellules de gestion de crise ainsi que les populations doivent dont anticiper les contraintes de l'épidémie en vue de cette évacuation massive. Cela passe par l'information aux populations, l'intégration des nouvelles contraintes dans les plans, l'adaptation des ressources mobilisables dans un contexte sanitaire potentiellement très dégradé, une prise en charge accrue des populations les plus vulnérables. Les incertitudes intrinsèques à toutes crises sont dans ce contexte multi risques démultipliées et nécessitent l'anticipation logistique et la préparation, tout en laissant une place à l'adaptation contextuelle.

La concordance entre pandémie de la COVID-19 et des événements majeurs déjà expérimentée dans plusieurs pays offre l'occasion aux acteurs de la gestion de crise, aux populations et aux chercheurs d'intégrer les contraintes multi risques à plusieurs échelles, du local au global. En effet les plans de gestion des risques sont trop souvent mono-aléa et circonscrits à l'intérieur d'un territoire restreint: le territoire à risque d'inondation, le territoire à risque volcanique, le territoire à risque cyclonique. Or il n'est pas rare qu'une catastrophe soit non seulement le résultat d'une capacité locale de gestion dépassée mais également d'une emprise spatiale de l'évènement disproportionnée par rapport à ce qui avait été anticipé. Ces deux enjeux sont saillants dans un contexte multi risques qui démultiplie les conditions de ruptures. La question des limites des systèmes de gestion de crise et des limites territoriales des crises anticipées permet alors d'imaginer de nouveaux scénarios de crises multi risques, et de s'y préparer.

\section{BIBLIOGRAPHIE}

Bercegol R. de, Goreau-Ponceaud A., Gowda S., Raj A., 2020. Confining the margins, marginalizing the confined: The Distress of Neglected Lockdown Victims in Indian Cities. EchoGéo [En ligne], Sur le Vif. URL: http://journals.openedition.org/echogeo/19357 - DOI: https://doi.org/10.4000/ echogeo.19357

Boyland M., Adelina C., 2020. COVID-19, Cyclone Amphan and building back better. Site web du Stockholm Environment Institute [En ligne]. URL : https://www.sei.org/perspectives/covid-19cyclone-amphan-and-building-back-better/

Bundy J., Pfarrer M.D., Cole E. 2017. Crises and Crisis Management: Integration, Interpretation, and Research Development. Journal of Management [En ligne], vol. 43, n 6, p. 1661-1692. DOI: https://doi.org/10.1177/0149206316680030

Canavesio R., Jeanson M., Étienne S., 2014. La gestion du risque cyclonique en Polynésie française et ses limites : exemple du cyclone tropical oli, février 2010. Bulletin de l'association de géographes français, vol. 91, n 3, p.325-337.

CEPRI, 2014. L'évacuation massive des populations : Les territoires face à l'inondation. Centre Européen de Prévention du Risque d'Inondation, 100 p.

Chittibabu P., Dube S.K., Macnabb J.B., Murty T. S., et al., 2004. Mitigation of Flooding and Cyclone Hazard in Orissa, India. Natural Hazards [En ligne], 31, p. 455-485. URL: https://link.springer.com/ 
article/10.1023/B:NHAZ.0000023362.26409.22 - DOI: https://doi.org/10.1023/B:NHAZ. 0000023362.26409 .22

Ćosić K., Popović S., Šarlija M., Kesedžić I., 2020. Impact of Human Disasters and COVID-19 Pandemic on Mental Health: Potential of Digital Psychiatry. Psychiatria Danubina [En ligne], vol. 32, $\mathrm{n}^{\circ}$ 1, p. : 25-31. URL: http://www.psychiatria-danubina.com/UserDocsImages/pdf/ dnb_vol32_no1/dnb_vol32_no1_25.pdf - DOI: https://doi.org/10.24869/psyd.2020.25

Daudé É. Chapuis K., Taillandier P., et al., 2019. ESCAPE: Exploring by Simulation Cities Awareness on Population Evacuation [En ligne]. ISCRAM, International Conference on Information Systems for Crisis Response and Management, p. 76-93. URL: https://halshs.archives-ouvertes.fr/ halshs-02144058

De Belizal E., Lavigne F., Gaillard J.-C., et al., 2012. The 2007 eruption of Kelut volcano (East Java, Indonesia): Phenomenology, crisis management and social response. Geomorphology, vol. 136, $\mathrm{n}^{\circ} 1$, p. 165-175.

Defossez S., Gherardi M., 2020. Face au cyclone Irma! Le rôle des populations dans la gestion de la crise à Saint-Martin (Petites Antilles, iles du Nord), EchoGéo [En ligne], n 51. URL: http:// journals.openedition.org/echogeo/18987 - DOI : https://doi.org/10.4000/echogeo.18987 Didier D., Bernatchez P., Dumont D., 2017. Systèmes d'alerte précoce pour les aléas naturels et environnementaux : virage ou mirage technologique? Revue des sciences de l'eau, vol. $30, \mathrm{n}^{\circ} 2$, p. 79-169.

Duanmu J, Taaffe K, Chowdhury M., 2010. Minimizing patient transport times during mass population evacuations. Transportation Research Record: Journal of the Transportation Research Board, vol. 2196, n 1, p. 150-158.

Dye C., Wolpert D.M, 1988. Earthquakes, influenza and cycles of Indian kala-azar. Transactions of the royal society of tropical medicine and hygiene, vol. 82, $n^{\circ}$ 6, p. 843-850.

Elysia V., Wihadanto A., 2018. The Sister Village Program: Promoting Community Resilience after Merapi Eruption. The Indonesian Journal of Planning and Development, vol. 3, n 1, P. 32-43.

Esposito Amideo A., Scaparra M.P., Kotiadis K., 2019. Optimising shelter location and evacuation routing operations: The critical issues. European Journal of Operational Research [En ligne], vol. 279, $\mathrm{n}^{\circ}$ 2, p. 279-295. DOI: https://doi.org/10.1016/j.ejor.2018.12.009

Few R., Matthies F., 2006. Flood Hazards and Health: Responding to Present and Future Risks. London, $212 \mathrm{p}$.

Floret N., Viel J.F., Mauny F., et al., 2006. Negligible Risk for Epidemics after Geophysical Disasters. Emerging Infectious Diseases [En ligne], vol. 2, n 4, p. 543-548. DOI: https://doi.org/10.3201/ eid1204.051569

Giese B. S., Slowey N. C., Ray S., et al., 2010. The 1918/19 El Niño. Bulletin of American Meteorological Society [En ligne], n 91, 177-183. DOI: https://doi.org/10.1175/2009BAMS2903.1

Guillet, S., Corona, C., Stoffel, M. et al., 2017. Climate response to the Samalas volcanic eruption in 1257 revealed by proxy records. Nature Geoscience [En ligne], n 10, p. 123-128. DOI: https:// doi.org/10.1038/ngeo2875

Gupta S.K., Suantio AA., Gray A., et al., 2007. Factors associated with E. Coli contamination of household drinking water among tsunami and earthquake survivors, Indonesia. Am. J. Trop. Med. Hyg., vol. 76, n 6, p. 1158-1162.

Janda, R.J., Daag, A.S., Delos Reyes, P.J., et al., 1996. Assessmentand response to lahar hazard around Mount Pinatubo, 1991-1993. In Newhall C.G., Punonbayan R.S. (ed.), Fire and mud: Eruptions 
and lahars of Mount Pinatubo. Philippines and Seattle, Quezon City, Philippine Institute of Volcanology and Seismology and University of Washington Press, p. 107-140.

Nikolich-Zugich J., Knox K.S., Tafich Rios C., Natt B., Bhattacharya D., Fain M.J., 2020. SARS-CoV-2 and COVID-19 in older adults: what we may expect regarding pathogenesis, immune responses, and outcomes. GeroScience [En ligne], n 42, p. 505-514. DOI: https://doi.org/10.1007/ s11357-020-00186-0

Jassempour K., Shirazi K.K., Fararooei M., et al., 2014. The impact of educational intervention for providing disaster survival kit: Applying precaution adoption process model. Int. J. Disaster Risk Reduct. [En ligne], n 10, p. 374-380. DOI: https://doi.org/10.1016/j.ijdrr.2014.10.012

Jenkins S., Lallemant D., Federico S., 2020. Pandemics \& Natural Hazards: The Potential Coincidence of a Very Contagious Disease with Another Disaster. Earth observatory blog [En ligne]. URL: https://earthobservatory.sg/blog/pandemics-natural-hazards-potential-coincidence-verycontagious-disease-another-disaster

Laliberté D., 2007. Crises humanitaires, santé des réfugiés et des déplacés : un cadre analytique. Revue européenne des migrations internationales, vol. 23, $\mathrm{n}^{\circ}$ 3, p. 85-96.

Lavigne F., Sahal A., 2011. La réalité des risques majeurs en Nouvelle-Calédonie. In Cheroux B., Faberon J. Y. (ed.), Risques majeurs et institutions en Nouvelle-Calédonie. Presses Universitaires d'AixMarseille, p. 33-55.

Lavigne F., Paris R., Grancher D., et al., 2009. Reconstruction of Tsunami Inland Propagation on December 26, 2004 in Banda Aceh, Indonesia, through Field Investigations. Pure and Applied Geophysics, n 166, p. 259-281, DOI: https://doi.org/10.1007/s00024-008-0431-8

Leone F., De Richemond N., Vinet F. 2010. Aléas naturels et gestion des risques. Presses Universitaires de France, 288 p.

Leone F., Komorowski J.-C., Gherardi-Leone M., et al., 2018. Accessibilité territoriale et gestion de crise volcanique aux Antilles françaises (Guadeloupe \& Martinique) : contribution à la planification des évacuations. Cybergeo : European Journal of Geography [En ligne], document 865, DOI: https://doi.org/10.4000/cybergeo.29425

Mei E.T.W., Lavigne F., 2013. Mass evacuation of the 2010 Merapi eruption. International Journal of Emergency Management, vol. 9, $\mathrm{n}^{\circ}$ 4, p. 298-311.

Moatty A., Grancher D., Virmoux C., Cavero J., 2019. Bilan humain de l'ouragan Irma à SaintMartin : la rumeur post-catastrophe comme révélateur des disparités socio-territoriales, Géocarrefour [En ligne], vol. 93, n 1. URL: http://journals.openedition.org/geocarrefour/12918 DOI: https://doi.org/10.4000/geocarrefour.12918

Omira R., Dogan G. Hidayat G., et al., 2019. The September 28th, 2018, Tsunami In Palu-Sulawesi, Indonesia: A Post-Event Field Survey. Pure and Applied Geophysics [En ligne], n ${ }^{\circ} 176$, p. 1379-1395. DOI: https://doi.org/10.1007/s00024-019-02145-z

Quigley M.C., Attanayake J., King A., Prideaux F., 2020. A multi-hazards earth science perspective on the COVID-19 pandemic: the potential for concurrent and cascading crises. Environ Syst Decis [En ligne], n 40, p. 199-215. DOI: https://doi.org/10.1007/s10669-020-09772-1

Organisation panaméricaine de la santé. 1982. L'hygiène du milieu après une catastrophe naturelle. Publication scientifique ${ }^{\circ}$ 430, 72 p., Accessible en ligne. URL: http://helid.digicollection.org/en/ d/J066f/5.html

Paul B.K., 2009. Why relatively fewer people died? The case of Bangladesh's Cyclone Sidr. Natural Hazards [En ligne], vol. 50, p. 289-304. DOI: https://doi.org/10.1007/s11069-008-9340-5 
Piarroux R., 2019. Choléra, Haïti 2000-2018, Histoire d'un désastre. Paris, Éditions du CNRS, 295 p.

Reghezza-Zitt M., 2019. Gestion de crise et incertitude(s) ou comment planifier le hors-cadre et l'inimaginable. Application aux crises résultant de crues majeures en Île-de-France Annales de géographie 2019/2 ( $\left.\mathrm{N}^{\circ} 726\right)$, p 5-30

Sauvagnargues S. (dir.), 2019. Prise de décision en situation de crise : recherche et innovations pour une formation optimale. ISTE Editions, $172 \mathrm{p}$.

Shaw R., Mallick F., Islam A. (ed.), 2013. Disaster Risk Reduction Approaches in Bangladesh. Springer, $366 \mathrm{p}$.

UN, 2020. Floods Response Plan, Viet Nam [En ligne]. 12 p. URL: https://reliefweb.int/sites/ reliefweb.int/files/resources/VNM-ResponsePlan-201031.pdf

UNESCO, 2020. Guide on Tsunami Evacuation during COVID-19, Jakarta [En ligne]. URL: https:// en.unesco.org/sites/default/files/guide_for_tsunami_evacuation_covid-1_final-1_eng.pdf

Valsecchi M., Durante R., 2020. Internal migration and the spread of COVID-19. New Economic School, Working Paper $n^{\circ} 276$ [En ligne], 44 p. URL: https://www.nes.ru/files/Preprints-resh/WP276.pdf Vinet F., 2018. La grande grippe. 1918. La pire épidémie du siècle. Ed. Vendémiaire, 256 p.

Widianto S., Christina B., Allard T., 2020. Indonesia, in major shift, to allow lockdowns as coronavirus cases soar. Site web de l'Agence Reuters [En ligne]. URL: https://www.reuters.com/ article/us-health-coronavirus-indonesia-lockdown-idUSKBN21E1LL

Zhang H., 2012. What has China Learnt from Disasters? Evolution of the Emergency Management System after SARS, Southern Snowstorm, and Wenchuan Earthquake. Journal of Comparative Policy Analysis: Research and Practice [En ligne], vol. 14, n 3, p. 234-244. DOI: https://doi.org/ 10.1080/13876988.2012.687621

\section{NOTES}

1. https://www.santepubliquefrance.fr/etudes-et-enquetes/covid-19-une-enquete-pour-suivre-levolution-des-comportements-et-de-la-sante-mentale-pendant-l-epidemie

2. https://www.dnc.nc/vanuatu-le-cyclone-harold-sinvite-en-pleine-crise-du-coronavirus

3. NDRF : http://www.ndrf.gov.in/

4. https://www.thenewhumanitarian.org/news-feature/2020/06/16/coronavirus-cyclonedisaster-risk-reduction-preparedness

5. https://www.huffingtonpost.fr/entry/apres-des-inondations-devastatrices-au-japon-450000personnes-contraintes-devacuer_fr_5f06b86fc5b6480493cb617f

6. https://www.thenewhumanitarian.org/news-feature/2020/06/16/coronavirus-cyclonedisaster-risk-reduction-preparedness

7. https://www.thejakartapost.com/seasia/2020/04/03/malaysias-indigenous-people-flee-intoforests-to-escape-coronavirus.html

8. https://earthjournalism.net/stories/no-space-in-shelters-as-severe-cyclone-nears-indiabangladesh

9. https://reliefweb.int/report/bangladesh/bangladesh-cyclone-final-report-early-actioneap2018bd01

10. https://www.youtube.com/watch?v=ALuiYitYg3s

11. https://www.huffingtonpost.fr/entry/apres-des-inondations-devastatrices-au-japon-450000personnes-contraintes-devacuer_fr_5f06b86fc5b6480493cb617f 
12. https://www.irdes.fr/recherche/2020/qes-249-les-inegalites-face-au-risque-de-detressepsychologique-pendant-le-confinement-premiers-resultats-enquete-coclico.html 13. https://www.lemonde.fr/big-browser/article/2020/03/23/covid-19-dans-le-monde-la-fievredu-stockage-et-une-ruee-sur-le-papier-toilette_6034158_4832693.html

14. https://www.miamiherald.com/news/weather/hurricane/article242864096.html

15. https://reliefweb.int/sites/reliefweb.int/files/resources/EAP2018BD01fr.pdf

\section{RÉSUMÉS}

Cet article propose une réflexion sur les contraintes que fait peser la focalisation de la gestion de la pandémie au niveau mondial et de l'épidémie au niveau des territoires sur la préparation et l'anticipation de nouvelles menaces, tels que des aléas naturels.

This paper offers a reflection on the constraints imposed by the focus of the management of the pandemic at the global level and of the epidemic at the level of the territories on the preparation and anticipation of new threats, such as natural hazards.

\section{INDEX}

Keywords : pandemic, multi-hazards, multi crisis, population evacuation

Mots-clés : pandémie, multi-aléa, multicrise, évacuation des populations

\section{AUTEURS}

\section{ÉRIC DAUDÉ}

Éric Daudé, eric.daude@cnrs.fr, est directeur de recherche au CNRS et membre de l'UMR IDEES. Il a récemment publié :

- Fenet J., Daudé É., 2020. La population, grande oubliée des politiques de prévention et de gestion territoriales des risques industriels : le cas de l'agglomération rouennaise. Cybergéo. European Journal of Geography [En ligne], document 932. URL: http://journals.openedition.org/cybergeo/ 34020 - DOI: https://doi.org/10.4000/cybergeo.34020

- Daudé É., Tranouez P., 2020. ESCAPE-SG : un simulateur d'évacuation massive de population pour la formation des acteurs à la gestion de crise. Netcom, Networks and Communication Studies [En ligne]. URL: http://journals.openedition.org/netcom/4340

- Daudé É., Chapuis K., Taillandier P., et al., 2019. ESCAPE: Exploring by Simulation Cities Awareness on Population Evacuation [En ligne]. Proceedings of the 16th ISCRAM Conference, Information Systems for Crisis Response and Management, p. 76-93. URL: https://halshs.archivesouvertes.fr/halshs-02144058

\section{DELPHINE GRANCHER}

Delphine Grancher, Delphine.GRANCHER@lgp.cnrs.fr, est ingénieur de recherche au Laboratoire de Géographie Physique (UMR 8591). Elle a récemment publié : 
- Moatty A., Grancher D., Virmoux C., Cavero J., 2020. Organisation de la post-catastrophe après Irma à Saint-Martin., EchoGéo [En ligne], n 51. URL: http://journals.openedition.org/echogeo/ 19017 - DOI: https://doi.org/10.4000/echogeo.19017

- Moatty A., Grancher D., Virmoux C., Cavero J.,2019. Bilan humain de l'ouragan Irma à SaintMartin : la rumeur post-catastrophe comme révélateur des disparités socio-territoriales., Géocarrefour [En ligne], vol. 93, n 1. URL: http://journals.openedition.org/geocarrefour/12918 DOI: https://doi.org/10.4000/geocarrefour.12918

- Oiry A., Grancher D., 2019. Le milieu scolaire : un terrain privilégié de l'enseignement et/ou de l'éducation aux risques naturels dans les territoires d'outre-mer? Les Cahiers d'Outre-Mer, $\mathrm{n}^{\circ} 280$, p. 567-598. URL: https://www.cairn.info/revue-les-cahiers-d-outre-mer-2019-2-page-567.htm

\section{FRANCK LAVIGNE}

Franck Lavigne, franck.lavigne@univ-paris1.fr, est professeur à l'Université Paris 1 PanthéonSorbonne/Institut Universitaire de France (IUF) et membre du Laboratoire de Géographie Physique. Il a récemment publié :

- Malawani M. N.., Lavigne F., Gomez C., Mutaqin B. W., Hadmoko D. S., 2021. Review of Local and Global Impacts of Volcanic Eruptions and Disaster Management Practices: The Indonesian Example. Geosciences [En ligne], vol. 11, n 3109. DOI: https://doi.org/10.3390/ geosciences11030109

- Meilianda E., Lavigne F., Pradhan B., et al., 2021. Barrier Islands Resilience to Extreme Events: Do Earthquake and Tsunami Play a Role? Water [En ligne], vol. 13, n². https://doi.org/10.3390/ w13020178

- Le Cozannet G., Oliveros C., Brivois O., et al., 2020. Detecting Changes in European Shoreline Evolution Trends Using Markov Chains and the Eurosion Database. Frontiers in Marine Science [En ligne], vol. 7. DOI: https://doi.org/10.3389/fmars.2020.00326 